\title{
On Ostrowski type inequalities via fractional integrals of a function with respect to another function
}

\section{Marian Matłoka}

Pozna University of Economics and Business al. Niepodległości 10, 61-875 Poznań, Poland.

\begin{abstract}
In this paper, we establish new Ostrowski type inequalities involving fractional integrals with respect to another function. Such fractional integrals generalize the Riemann-Liouville fractional integrals and the Ostrowski type fractional integrals.
\end{abstract}

Keywords: Ostrowski type inequality, h-convex function, fractional integral.

2010 MSC: 26D15, 26A51, 26A33, 49J40, 90C33.

(C)2020 All rights reserved.

\section{Introduction}

The following Ostrowski inequality is well known [4]:

$$
\left|f(x)-\frac{1}{b-a} \int_{a}^{b} f(t) d t\right| \leqslant\left[\frac{1}{4}+\frac{\left(x-\frac{a+b}{2}\right)^{2}}{(b-a)^{2}}\right] M(b-a) .
$$

Ostrowski proved this inequality in 1938, and since then it has been generalized in a number of ways (see $[1-3,5,7,9])$.

In [10], the class of functions which are h-convex has been introduced by Varošanec as the following.

Definition 1.1. A function $f: I \rightarrow R, \theta \neq I \subset R$ being an interval, is called h-convex if

$$
f(t x+(1-t) y) \leqslant h(t) f(x)+h(1-t) f(y)
$$

holds for all $x, y \in I, t \in(0,1)$, where $h: J \rightarrow R, h \neq 0$ and $J$ is an interval, $(0,1) \subseteq J$.

In [8], Sarikaya et al. proved that for h-convex function the following variant of the Hadamard inequality is fulfilled:

$$
\frac{1}{2 h\left(\frac{1}{2}\right)} f\left(\frac{a+b}{2}\right) \leqslant \frac{1}{b-a} \int_{a}^{b} f(x) d x \leqslant[f(a)+f(b)] \int_{0}^{1} h(t) d t .
$$

Email address: marian.matloka@ue.poznan.pl (Marian Matłoka)

doi: $10.22436 /$ jnsa.013.02.04

Received: 2019-07-17 Revised: 2019-08-06 Accepted: 2018-08-31 
The aim of this paper is to establish new Ostrowski inequalities for h-convex functions involving fractional integrals with respect to another function. The obtained results generalize some existing results from the literature.

First, we give some necessary definitions of fractional calculus theory that will be used through this paper. For more details, one can consult [6].

Definition 1.2. Let $f \in L([a, b])$. The Riemann-Liouville integrals $I_{a^{+}}^{\alpha} f$ and $I_{b^{-}}^{\alpha} f$ of order $\alpha>0$ with $a \geqslant 0$ are defined by

$$
\mathrm{I}_{a^{+}}^{\alpha} f(x)=\frac{1}{\Gamma(\alpha)} \int_{a}^{x}(x-t)^{\alpha-1} f(t) d t, x>a \quad \text { and } \quad I_{b^{-}}^{\alpha} f(x)=\frac{1}{\Gamma(\alpha)} \int_{x}^{b}(t-x)^{\alpha-1} f(t) d t, x<b,
$$

respectively, where $\Gamma(\alpha)$ is the Gamma function. Here $I_{a^{+}}^{\alpha} f(x)=I_{b^{-}}^{\alpha} f(x)=f(x)$.

In the case of $\alpha=1$, the fractional integral reduces to the classical integral.

Definition 1.3. Let $g:[a, b] \rightarrow R$ be an increasing and positive function on $(a, b]$, having a continuous derivative $g^{\prime}(x)$ on $(a, b)$. The fractional integrals $I_{a^{+}, g}^{\alpha} f$ and $I_{b^{-}, g}^{\alpha} f$ of $f$ with respect to the function $g$ on $[a, b]$ of order $\alpha>0$ are defined by

$$
I_{a^{+}, g}^{\alpha} f(x)=\frac{1}{\Gamma(\alpha)} \int_{a}^{x}(g(x)-g(t))^{\alpha-1} g^{\prime}(t) f(t) d t, x>a
$$

and

$$
\mathrm{I}_{\mathrm{b}^{-}, \mathrm{g}}^{\alpha} f(x)=\frac{1}{\Gamma(\alpha)} \int_{x}^{\mathrm{b}}(g(t)-g(x))^{\alpha-1} g^{\prime}(t) f(t) d t, x<b
$$

respectively.

Observe that for $g(x)=x$ the above fractional integrals reduce to the Riemann-Liouville fractional integrals.

Throughout this paper, we will assume that $g:[a, b] \rightarrow R$ is an increasing and positive function on $[a, b]$, having a continuous derivative $g^{\prime}(x)$ on $(a, b)$.

\section{Main results}

In order to prove our main theorems, we need the following Lemma.

Lemma 2.1. Let $\mathrm{f}:[\mathrm{a}, \mathrm{b}] \rightarrow \mathrm{R}$ be a differentiable mapping on $(\mathrm{a}, \mathrm{b})$ with $\mathrm{a}<\mathrm{b}, \mathrm{f}^{\prime}:[\mathrm{a}, \mathrm{b}] \rightarrow \mathrm{R}$ be integrable on $[a, b]$, and $x \in(a, b)$. Then the following equality holds:

$$
\begin{aligned}
f(x)-\Gamma(\alpha+1)\left[\frac{1}{2(g(b)-g(x))^{\alpha}} I_{x^{+}, g}^{\alpha} f(b)+\frac{1}{2(g(x)-g(a))^{\alpha}} I_{x^{-}, g}^{\alpha} f(a)\right] \\
=\frac{x-a}{2(g(x)-g(a))^{\alpha}} \int_{0}^{1}(g(t x+(1-t) a)-g(a))^{\alpha} f^{\prime}(t x+(1-t) a) d t \\
\quad-\frac{b-x}{2(g(b)-g(x))^{\alpha}} \int_{0}^{1}(g(b)-g(t x+(1-t) b))^{\alpha} f^{\prime}(t x+(1-t) b) d t .
\end{aligned}
$$

Proof. By integration by parts and changing the variables, we get

$$
I_{1}=\int_{0}^{1}(g(t x+(1-t) a)-g(a))^{\alpha} f^{\prime}(t x+(1-t) a) d t
$$




$$
\begin{aligned}
= & \left.\frac{1}{x-a}(g(t x+(1-t) a)-g(a))^{\alpha} f(t x+(1-t) a)\right|_{0} ^{1} \\
& -\alpha \int_{0}^{1}(g(t x+(1-t) a)-g(a))^{\alpha-1} g^{\prime}(t x+(1-t) a) f(t x+(1-t) a) d t \\
= & \frac{1}{x-a}\left\{(g(x)-g(a))^{\alpha} f(x)-\alpha \int_{a}^{x}(g(u)-g(a))^{\alpha-1} g^{\prime}(u) f(u) d u\right\} \\
= & \frac{1}{x-a}\left\{(g(x)-g(a))^{\alpha} f(x)-\Gamma(\alpha+1) I_{x^{-}, g}^{\alpha} f(a)\right\}
\end{aligned}
$$

and similarly

$$
\begin{aligned}
I_{2}= & \int_{0}^{1}(g(b)-g(t x+(1-t) b))^{\alpha} f^{\prime}(t x+(1-t) b) d t \\
= & \left.\frac{1}{x-b}(g(b)-g(t x+(1-t) b))^{\alpha} f(t x+(1-t) b)\right|_{0} ^{1} \\
& +\alpha \int_{0}^{1}(g(b)-g(t x+(1-t) b))^{\alpha-1} g^{\prime}(t x+(1-t) b) f(t x+(1-t) b) d t \\
= & -\frac{1}{b-x}\left\{(g(b)-g(x))^{\alpha} f(x)-\alpha \int_{x}^{b}(g(b)-g(u))^{\alpha-1} g^{\prime}(u) f(u) d u\right\} \\
= & -\frac{1}{b-x}\left\{(g(b)-g(x))^{\alpha} f(x)-\Gamma(\alpha+1) I_{x^{+}, g}^{\alpha} f(x)\right\} .
\end{aligned}
$$

Thus, we can write

$$
\begin{aligned}
f(x) & -\Gamma(\alpha+1)\left[\frac{1}{2(g(b)-g(x))^{\alpha}} I_{x^{+}, g}^{\alpha} f(b)+\frac{1}{2(g(x)-g(a))^{\alpha}} I_{x^{-}, g}^{\alpha} f(a)\right] \\
& =\frac{x-a}{2(g(x)-g(a))^{\alpha}} I_{1}-\frac{b-x}{2(g(b)-g(x))^{\alpha}} I_{2}
\end{aligned}
$$

which completes the proof.

Throughout this paper, let

$$
I(f, g, x, a, b)=f(x)-\Gamma(\alpha+1)\left[\frac{1}{2(g(b)-g(x))^{\alpha}} I_{x^{+}, g}^{\alpha} f(b)+\frac{1}{2(g(x)-g(a))^{\alpha}} I_{x^{-}, g}^{\alpha} f(a)\right] .
$$

Now, we are ready to state and prove our results.

Theorem 2.2. Suppose that all assumptions of Lemma 2.1 hold. Additionally, assume that $\left|f^{\prime}\right|$ is h-convex on $[a, b]$ and $\left|f^{\prime}(x)\right| \leqslant M,\left|g^{\prime}(x)\right| \leqslant L, x \in[a, b]$. Then for each $x \in(a, b)$ the following inequality holds:

$$
|I(f, g, x, a, b)| \leqslant\left[\frac{(x-a)^{\alpha+1}}{2(g(x)-g(a))^{\alpha}}+\frac{(b-x)^{\alpha+1}}{2(g(b)-g(x))^{\alpha}}\right] M L^{\alpha} \int_{0}^{1} t^{\alpha}(h(t)+h(1-t)) d t .
$$

Proof. From Lemma 2.1 we have

$$
\begin{aligned}
|I(f, g, x, a, b)| \leqslant & \frac{x-a}{2(g(x)-g(a))^{\alpha}} \int_{0}^{1}(g(t x+(1-t) a)-g(a))^{\alpha}\left|f^{\prime}(t x+(1-t) a)\right| d t \\
& +\frac{b-x}{2(g(b)-g(x))^{\alpha}} \int_{0}^{1}(g(b)-g(t x+(1-t) b))^{\alpha}\left|f^{\prime}(t x+(1-t) b)\right| d t .
\end{aligned}
$$


Since $g$ is differentiable and $\left|g^{\prime}(x)\right| \leqslant L$ on $[a, b]$, we get that $g$ is the Lipschitzian function. This means that

$$
g(t x+(1-t) a)-g(a) \leqslant L t(x-a) \text { and } g(b)-g(t x+(1-t) b) \leqslant L t(b-x) .
$$

Since $\left|f^{\prime}\right|$ is h-convex on $[a, b]$ and $\left|f^{\prime}(x)\right| \leqslant M$, we get

$$
\begin{aligned}
& \int_{0}^{1}(g(t x+(1-t) a)-g(a))^{\alpha}\left|f^{\prime}(t x+(1-t) a)\right| d t \\
& \quad \leqslant L^{\alpha}(x-a)^{\alpha} \int_{0}^{1} t^{\alpha}\left(\left|f^{\prime}(x)\right| h(t)+\left|f^{\prime}(a)\right| h(1-t)\right) d t \leqslant L^{\alpha}(x-a)^{\alpha} M \int_{0}^{1} t^{\alpha}(h(t)+h(1-t)) d t
\end{aligned}
$$

and similarly

$$
\begin{aligned}
& \int_{0}^{1}(g(b)-g(t x+(1-t) b))^{\alpha}\left|f^{\prime}(t x+(1-t) b)\right| d t \\
& \quad \leqslant L^{\alpha}(b-x)^{\alpha} \int_{0}^{1} t^{\alpha}\left(\left|f^{\prime}(x)\right| h(t)+\left|f^{\prime}(b)\right| h(1-t)\right) d t \leqslant L^{\alpha}(b-x)^{\alpha} M \int_{0}^{1} t^{\alpha}(h(t)+h(1-t)) d t .
\end{aligned}
$$

Hence, we have

$$
|I(f, g, x, a, b)| \leqslant\left[\frac{(x-a)^{\alpha+1}}{2(g(x)-g(a))^{\alpha}}+\frac{(b-x)^{\alpha+1}}{2(g(b)-g(x))^{\alpha}}\right] M L^{\alpha} \int_{0}^{1} t^{\alpha}(h(t)+h(1-t)) d t,
$$

which completes the proof.

Corollary 2.3. In Theorem 2.2, if we take $\mathrm{h}(\mathrm{t})=\mathrm{t}$, the inequality (2.1) becomes the following inequality for convex function:

$$
|I(f, g, x, a, b)| \leqslant\left[\frac{(x-a)^{\alpha+1}}{2(g(x)-g(a))^{\alpha}}+\frac{(b-x)^{\alpha+1}}{2(g(b)-g(x))^{\alpha}}\right] \frac{M L^{\alpha}}{(\alpha+1)} .
$$

Corollary 2.4. In Theorem 2.2, if we take $\mathrm{h}(\mathrm{t})=\mathrm{t}^{\mathrm{s}}, \mathrm{s} \in(0,1]$, then inequality (2.1) becomes the following inequality for s-convex functions:

$$
|I(f, g, x, a, b)| \leqslant\left[\frac{(x-a)^{\alpha+1}}{2(g(x)-g(a))^{\alpha}}+\frac{(b-x)^{\alpha+1}}{2(g(b)-g(x))^{\alpha}}\right] M L^{\alpha}\left[\frac{1}{\alpha+s+1}+\frac{\Gamma(\alpha+1) \Gamma(s+1)}{\Gamma(\alpha+s+2)}\right] .
$$

Remark 2.5. In Theorem (2.2), if we choose $g(x)=x$, then inequality (2.1) becomes the inequality 2.2 of Theorem 1 in [3].

Theorem 2.6. Suppose that all assumptions of Lemma 2.1 hold. Additionally, assume that $\left|\mathrm{f}^{\prime}\right|^{\mathrm{q}}$ is h-convex on $[\mathrm{a}, \mathrm{b}], \mathrm{q}>1$, and $\left|\mathrm{f}^{\prime}(\mathrm{x})\right| \leqslant M,\left|\mathrm{~g}^{\prime}(\mathrm{x})\right| \leqslant \mathrm{L}, \mathrm{x} \in[\mathrm{a}, \mathrm{b}]$. Then for each $\mathrm{x} \in(\mathrm{a}, \mathrm{b})$ the following inequality holds:

$$
|I(f, g, x, a, b)| \leqslant\left[\frac{(x-a)^{\alpha+1}}{2(g(x)-g(a))^{\alpha}}+\frac{(b-x)^{\alpha+1}}{2(g(b)-g(x))^{\alpha}}\right] \frac{M L^{\alpha}}{(\alpha p+1)^{\frac{1}{p}}}\left(2 \int_{0}^{1} h(t) d t\right)^{\frac{1}{q}},
$$

where $\frac{1}{\mathrm{p}}+\frac{1}{\mathrm{q}}=1$.

Proof. From Lemma 2.1 and using the well known Hölder inequality, we have

$$
|I(f, g, x, a, b)| \leqslant L^{\alpha} \frac{(x-a)^{\alpha+1}}{2(g(x)-g(a))^{\alpha}} \int_{0}^{1} t^{\alpha}\left|f^{\prime}(t x+(1-t) a)\right| d t
$$




$$
\begin{aligned}
& \quad+L^{\alpha} \frac{(b-x)^{\alpha+1}}{2(g(b)-g(x))^{\alpha}} \int_{0}^{1} t^{\alpha}\left|f^{\prime}(t x+(1-t) b)\right| d t \\
& \leqslant L^{\alpha} \frac{(x-a)^{\alpha+1}}{2(g(x)-g(a))^{\alpha}}\left(\int_{0}^{1} t^{\alpha p} d t\right)^{\frac{1}{p}}\left(\int_{0}^{1}\left|f^{\prime}(t x+(1-t) a)\right|^{q} d t\right)^{\frac{1}{q}} \\
& \quad+L^{\alpha} \frac{(b-x)^{\alpha+1}}{2(g(b)-g(x))^{\alpha}}\left(\int_{0}^{1} t^{\alpha p} d t\right)^{\frac{1}{p}}\left(\int_{0}^{1}\left|f^{\prime}(t x+(1-t) b)\right|^{q} d t\right)^{\frac{1}{q}} .
\end{aligned}
$$

Since $\left|f^{\prime}\right|^{q}$ is h-convex on $[a, b]$ and $\left|f^{\prime}(x)\right| \leqslant M$, we get

$$
\int_{0}^{1}\left|f^{\prime}(t x+(1-t) a)\right|^{q} d t \leqslant 2 M^{q} \int_{0}^{1} h(t) d t \quad \text { and } \int_{0}^{1}\left|f^{\prime}(t x+(1-t) b)\right|^{q} d t \leqslant 2 M^{q} \int_{0}^{1} h(t) d t
$$

and by simple computation

$$
\int_{0}^{1} t^{\alpha p} d t=\frac{1}{\alpha p+1}
$$

Hence, we have

$$
|I(f, g, x, a, b)| \leqslant\left[\frac{(x-a)^{\alpha+1}}{2(g(x)-g(a))^{\alpha}}+\frac{(b-x)^{\alpha+1}}{2(g(b)-g(x))^{\alpha}}\right] \frac{M L^{\alpha}}{(\alpha p+1)^{\frac{1}{p}}}\left(2 \int_{0}^{1} h(t) d t\right)^{\frac{1}{q}},
$$

which completes the proof.

Remark 2.7. In Theorem 2.6, if we choose $g(x)=x$, then inequality (2.2) becomes the inequality 2.6 of Theorem 2 in [3].

Theorem 2.8. Suppose that all assumptions of Lemma 2.1 hold. Additionally, assume that $\left|f^{\prime}\right|^{\mathrm{q}}$ is $h$-convex on $[a, b], q \geqslant 1$, and $\left|f^{\prime}(x)\right| \leqslant M,\left|g^{\prime}(x)\right| \leqslant L, x \in[a, b]$. Then for each $x \in(a, b)$ the following inequality holds:

$$
\begin{aligned}
& |\mathrm{I}(f, g, x, a, b)| \\
& \quad \leqslant\left[\frac{(x-a)^{\alpha+1}}{2(g(x)-g(a))^{\alpha}}+\frac{(b-x)^{\alpha+1}}{2(g(b)-g(x))^{\alpha}}\right] M L^{\alpha}\left(\frac{1}{\alpha+1}\right)^{1-\frac{1}{q}}\left(\int_{0}^{1} t^{\alpha}(h(t)+h(1-t)) d t\right)^{\frac{1}{q}} .
\end{aligned}
$$

Proof. From Lemma 2.1 and using the well known power mean inequality, we have

$$
\begin{aligned}
|I(f, g, x, a, b)| \leqslant & L^{\alpha} \frac{(x-a)^{\alpha+1}}{2(g(x)-g(a))^{\alpha}} \int_{0}^{1} t^{\alpha}\left|f^{\prime}(t x+(1-t) a)\right| d t \\
& +L^{\alpha} \frac{(b-x)^{\alpha+1}}{2(g(b)-g(x))^{\alpha}} \int_{0}^{1} t^{\alpha}\left|f^{\prime}(t x+(1-t) b)\right| d t \\
\leqslant & L^{\alpha} \frac{(x-a)^{\alpha+1}}{2(g(x)-g(a))^{\alpha}}\left(\int_{0}^{1} t^{\alpha} d t\right)^{1-\frac{1}{q}}\left(\int_{0}^{1} t^{\alpha}\left|f^{\prime}(t x+(1-t) a)\right|^{q} d t\right)^{\frac{1}{q}} \\
& +L^{\alpha} \frac{(b-x)^{\alpha+1}}{2(g(b)-g(x))^{\alpha}}\left(\int_{0}^{1} t^{\alpha} d t\right)^{1-\frac{1}{q}}\left(\int_{0}^{1} t^{\alpha}\left|f^{\prime}(t x+(1-t) b)\right|^{q} d t\right)^{\frac{1}{q}} .
\end{aligned}
$$

Since $\left|f^{\prime}\right|^{q}$ is h-convex on $[a, b]$ and $\left|f^{\prime}(x)\right| \leqslant M$, we get

$$
\int_{0}^{1} t^{\alpha}\left|f^{\prime}(t x+(1-t) a)\right|^{q} d t \leqslant M^{q} \int_{0}^{1} t^{\alpha}(h(t)+h(1-t)) d t
$$


and

$$
\int_{0}^{1} t^{\alpha}\left|f^{\prime}(t x+(1-t) b)\right|^{q} d t \leqslant M^{q} \int_{0}^{1} t^{\alpha}(h(t)+h(1-t)) d t
$$

Hence, we have

$$
\begin{aligned}
& |I(f, g, x, a, b)| \\
& \quad \leqslant\left[\frac{(x-a)^{\alpha+1}}{2(g(x)-g(a))^{\alpha}}+\frac{(b-x)^{\alpha+1}}{2(g(b)-g(x))^{\alpha}}\right] M L^{\alpha}\left(\frac{1}{\alpha+1}\right)^{1-\frac{1}{q}}\left(\int_{0}^{1} t^{\alpha}(h(t)+h(1-t)) d t\right)^{\frac{1}{q}},
\end{aligned}
$$

which completes the proof.

Remark 2.9. In Theorem 2.8, if we choose $g(x)=x$, then inequality (2.3) becomes the inequality 2.10 of Theorem 3 in [3].

Theorem 2.10. Suppose that all assumptions of Lemma 2.1 hold. Additionally, assume that $\left|\mathrm{f}^{\prime}\right|^{\mathrm{q}}$ is h-convex on $[\mathrm{a}, \mathrm{b}], \mathrm{q}>1$, and $\left|\mathrm{f}^{\prime}(\mathrm{x})\right| \leqslant \mathrm{M},\left|\mathrm{g}^{\prime}(\mathrm{x})\right| \leqslant \mathrm{L}, \mathrm{x} \in[\mathrm{a}, \mathrm{b}]$. Then for each $\mathrm{x} \in(\mathrm{a}, \mathrm{b})$ the following inequality holds:

$$
|I(f, g, x, a, b)| \leqslant\left[\frac{(x-a)^{\alpha+1}}{2(g(x)-g(a))^{\alpha}}+\frac{(b-x)^{\alpha+1}}{2(g(b)-g(x))^{\alpha}}\right] 2 L^{\alpha} M^{q}\left(\frac{1}{\alpha p+1}\right)^{\frac{1}{p}}\left(\int_{0}^{1} h(t) d t\right)^{\frac{1}{q}}
$$

where $\frac{1}{\mathrm{p}}+\frac{1}{\mathrm{q}}=1$.

Proof. From Lemma 2.1 and using the Hölder inequality, we have

$$
\begin{aligned}
|I(f, g, x, a, b)| \leqslant & L^{\alpha} \frac{(x-a)^{\alpha+1}}{2(g(x)-g(a))^{\alpha}}\left(\int_{0}^{1} t^{\alpha p} d t\right)^{\frac{1}{p}}\left(\int_{0}^{1}\left|f^{\prime}(t x+(1-t) a)\right|^{q} d t\right)^{\frac{1}{q}} \\
& +L^{\alpha} \frac{(b-x)^{\alpha+1}}{2(g(b)-g(x))^{\alpha}}\left(\int_{0}^{1} t^{\alpha p} d t\right)^{\frac{1}{p}}\left(\int_{0}^{1}\left|f^{\prime}(t x+(1-t) b)\right|^{q} d t\right)^{\frac{1}{q}} .
\end{aligned}
$$

Since $\left|f^{\prime}\right|^{q}$ is h-convex, by (1.1) we have

$$
\int_{0}^{1}\left|f^{\prime}(t x+(1-t) a)\right|^{q} d t=\frac{1}{x-a} \int_{a}^{x}\left|f^{\prime}(u)\right|^{q} d u \leqslant\left[\left|f^{\prime}(x)\right|^{q}+\left|f^{\prime}(a)\right|^{q}\right] \int_{0}^{1} h(t) d t
$$

and

$$
\int_{0}^{1}\left|f^{\prime}(t x+(1-t) b)\right|^{q} d t=\frac{1}{b-x} \int_{x}^{b}\left|f^{\prime}(u)\right|^{q} d u \leqslant\left[\left|f^{\prime}(x)\right|^{q} d u+\left|f^{\prime}(b)\right|^{q}\right] \int_{0}^{1} h(t) d t .
$$

Therefore we obtain

$$
\begin{aligned}
|I(f, g, x, a, b)| \leqslant & {\left[L^{\alpha} \frac{(x-a)^{\alpha+1}}{2(g(x)-g(a))^{\alpha}}\left(\left|f^{\prime}(x)\right|^{q}+\left|f^{\prime}(a)\right|^{q}\right)\right.} \\
& \left.+L^{\alpha} \frac{(b-x)^{\alpha+1}}{2(g(b)-g(x))^{\alpha}}\left(\left|f^{\prime}(x)\right|^{q}+\left|f^{\prime}(b)\right|^{q}\right)\right]\left(\frac{1}{\alpha p+1}\right)^{\frac{1}{p}}\left(\int_{0}^{1} h(t) d t\right)^{\frac{1}{q}} \\
\leqslant & {\left[\frac{(x-a)^{\alpha+1}}{2(g(x)-g(a))^{\alpha}}+\frac{(b-x)^{\alpha+1}}{2(g(b)-g(x))^{\alpha}}\right] 2 L^{\alpha} M^{q}\left(\frac{1}{\alpha p+1}\right)^{\frac{1}{p}}\left(\int_{0}^{1} h(t) d t\right)^{\frac{1}{q}}, }
\end{aligned}
$$

which completes the proof.

Remark 2.11. If we choose $h(t)=t$ or $h(t)=t^{s}, s \in(0,1]$ in Theorems 2.6, 2.8, and 2.10, we obtain the inequalities for convex or s-convex functions, respectively. 


\section{References}

[1] A. O. Akdemir, M. Tunç, Ostrowski type inequalities for s-logarithmically convex functions in the second sense with applications, Georgian Math. J., 22 (2015), 1-7. 1

[2] M. Alomari, M. Darus, S. S. Dragomir, P. Cerone, Ostrowski type inequalities for functions whose derivatives are s-convex in the second sense, Appl. Math. Lett., 23 (2010), 1071-1076.

[3] M. Matłoka, Ostrowski type inequalities for functions whose derivatives are h-convex via fractional integrals, J. Scient. Res. Rep., 3 (2014), 1633-1641. 1, 2.5, 2.7, 2.9

[4] A. Ostrowski, Uber die absolutabweichung einer differentierbaren Funktion von ihren Integralmittelwert, Comment. Math. Helv., 10 (1938), 226-227. 1

[5] M. E. Özdemir, C. Yildiz, An Ostrowski type ineequality for derivatives of q-th power of s-convex functions via fractional integrals, Georgian Math. J., 21 (2014), 491-498. 1

[6] S. G. Samko, A. A. Kilbas, O. I. Marichev, Fractional Integrals and Derivatives: Theory and Applications, Gordon and Breach, New York, (1993). 1

[7] M. Z. Sarikaya, On the Ostrowski type integral inequality, Acta Math. Univ. Comenian (N. S.), 79 (2010), 129-134. 1

[8] M. Z. Sarikaya, A. Saglam, H. Yildirim, On some Hadamard-type inequalities for h-convex functions, J. Math. Inequal., 2 (2008), 335-341. 1

[9] E. Set, New inequalities of Ostrowski type for mappings whose derivatives are s-convex in the second sense via fracional integrals, Comput. Math. Appl., 63 (2012), 1147-1154. 1

[10] S. Varošanec, On h-convexity, J. Math. Anal. Appl., 326 (2007), 303-311. 1 